\title{
Clinical manifestations of familial 13;18 translocation
}

\author{
WILLIAM A BLATTNER*, MILDRED L KISTENMACHER†, \\ SHIEN TSAI + , HOPE H PUNNETT $\dagger$, AND ELOISE R GIBLETT \\ From * the Environmental Epidemiology Branch, National Cancer Institute, Bethesda, Maryland 20205; \\ †St Christopher's Hospital for Children, Philadelphia, Pennsylvania 19133; \\ $\ddagger$ Biotech Research Laboratories Inc, Rockville, Maryland 20852; and §University of Washington, \\ School of Medicine, King County Central Blood Bank, Seattle, Washington 98104, USA
}

SUMMARY Female first cousins, aged 21 and $2 \frac{1}{2}$ years, with many of the characteristic features of trisomy 18 , were found to have identical unbalanced translocations, $46, X X,-13,+\operatorname{der}(13) t(13 ; 18)$ (p13;q12)mat. Clinical features of another cousin, two uncles, and an aunt suggested that they, too, had a partial trisomy 18 phenotype. The long survival and normal menstrual and secondary sexual development in one case are remarkable. A heritable balanced translocation, $46, \mathrm{XX}$ or $\mathrm{XY}, \mathrm{t}(13 ; 18)$ (p13;q12), was detected in the mothers of the cases, a sib, an aunt, and two uncles. Translocation carriers had abnormalities in gonadal structure or function, with aspermia in males and polycystic ovaries with infertility in several females, suggesting that some gene controlling reproductive development occurs on the long arm of chromosome 18 , with normal function interrupted at the breakpoint. Balanced translocation carriers may also be at greater risk for both benign and malignant neoplasms, which included acute leukaemia in an uncle and adenocarcinoma of the stomach at an early age in the grandmother. Although aetiological laboratory studies identified no premalignant state, the clinical findings suggest a defect that may predispose to cytogenetic abnormalities and malignancy.

Trisomy 18 is a well-characterised cytogenetic syndrome with an incidence in newborns of $0.01 \%$. In rare instances, the trisomy 18 phenotype results from an unbalanced translocation, arising as a primary mutation or through inheritance from a parent with a balanced translocation. ${ }^{2}$ In the present family (fig 1), female first cousins had partial trisomy 18 phenotype because they inherited identical $13 p+$ chromosomes from their mothers, who carried a balanced translocation of the long arm of chromosome 18 onto the short arm of chromosome 13 . Some balanced translocation carriers over three generations experienced abnormalities of reproductive function, as well as possible heightened susceptibility to neoplasia.

\section{Materials and methods}

Members of the family were interviewed and biological specimens obtained whth informed consent between 1975 and 1977. Hospital and vital records were obtained when possible to confirm medical diagnoses.

Routine haematological tests were performed by

Received for publication 6 November 1979 the National Institutes of Health Clinical Center. Quantitative immunoglobulins, immunoglobulin electrophoresis, autoantibodies, and gonadotrophins in males were measured on serum samples using standard techniques (Bioscience Laboratories, Van Nuys, California). Red blood cell antigens were tested by the NIH Blood Bank, red blood cell enzymes were assayed by electrophoretic methods, ${ }^{3}$ and HLA-A, -B, and $-C$ antigens were determined serologically. ${ }^{4}$ In vitro response to standard mitogens and pooled allogeneic cells in one-way mixed leucocyte culture was quantified by the relative proliferation index. ${ }^{5}$

Chromosome analysis was performed from phytohaemagglutinin stimulated peripheral lymphocytes and, in addition to conventional Giemsa staining, chromosome preparations were processed for $\mathbf{G}$ bands by trypsin treatment, ${ }^{6} 7$ for $\mathbf{R}$ bands, ${ }^{8}$ for $\mathbf{C}$ bands, ${ }^{9}$ and for $\mathrm{N}$ bands by a simplified silver staining method. ${ }^{10}$

\section{Case reports}

The proband (III.8) (fig 2a) was born in 1974 to unrelated 29-year-old parents. Because of fertility 


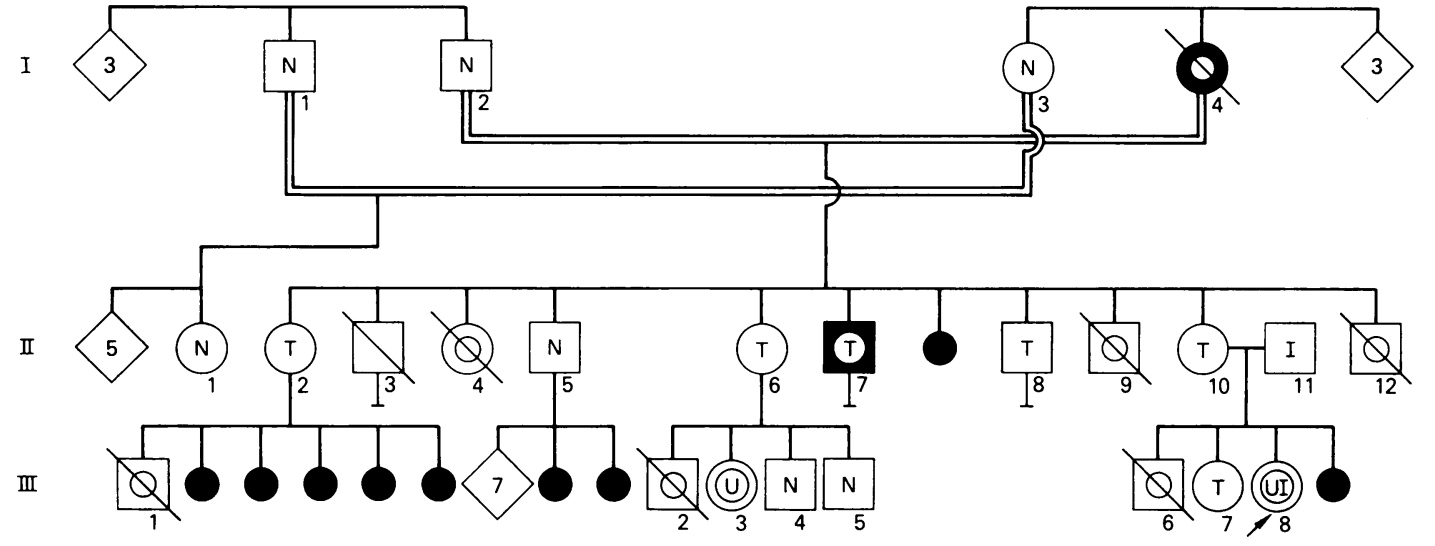

DO O

(T) Balanced translocation; $46, x x, t(13 ; 18)(p 13 ; q 12)$

(O) Clinically abnormal, not karyotyped

Mis carriage 0

Three clinically normal
males or females

N Clinically normal with normal karyotype
(U) Clinically abnormal with unbalanced translocati
$46, X X$, der $(13), t(13 ; 18)$ ( $p 13 ; q 12$ ) mat

(I) Carrier of pericentric $(p 11 ; q 12)$

FIG 1 Family pedigree.

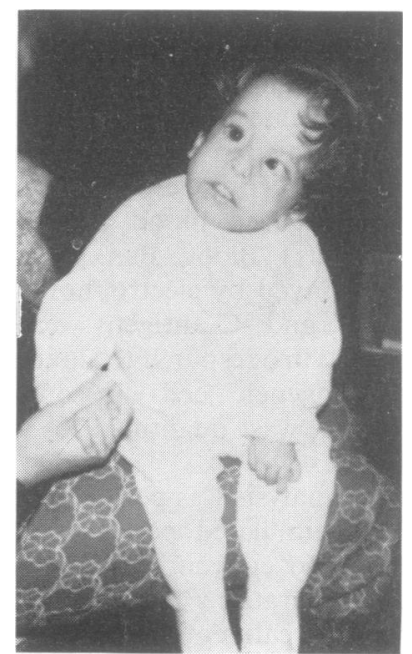

FIG 2 (a) Proband (III.8) at 3 years of age.

problems, the mother took prednisone for the first two weeks after conception. The vaginal delivery of a footling breech at 38 weeks was complicated by complete placental separation, and severe acidosis, muscular hypotonia, and grand mal seizures during $\frac{2}{\mathrm{D}}$ resuscitation. At 5 months, on referral to $\mathrm{St}$ Christopher's Hospital for Children (SCHC), she was found to have partial trisomy 18 phenotype, with microcephaly, low set ears, high arched palate, smale mandible, alternating esotropia, close set eyes, congenitally blocked tear ducts, pectus excavatum partial syndactyly of the second and third toes, $\infty$ haemangioma of the second toe of the right foot 3 . spasticity of all extremities, tight hips with difficuli abduction, hypoplastic labia majora, large clefe clitoris, and a ventricular septal defect. Dermatoo glyphs showed six arches and four very simple, low? count loops. At 5 years of age, the child is severelyo retarded and requires total supportive care.

A first cousin of the proband (III.3) (fig 2b) waș born one month postmature in 1956 to a 21 -year-old mother and 26-year-old father. She experienced difficulty in feeding as an infant and had delaye of developmental milestones. At $2 \frac{1}{2}$ years of age a persistent ductus arteriosus was repaired. At 22 years? of age, she is a severely retarded, spastic quadri plegic who weighs $31.8 \mathrm{~kg}$. Morphological abnor malities include flexion contractures of elbows and knees, distal shortening of upper and lower extremis. ties, overlap of fingers, and valgus deformity of feet 


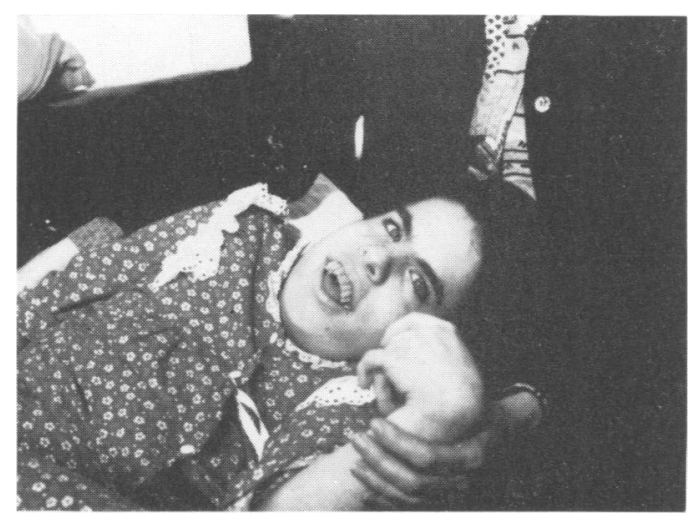

FIG 2 (b) First cousin of proband (III.3) at 21 years of age.

She has internal strabismus, high arched and narrow palate, an open bite with prominent upper central incisors, and hypertrophied gingivae. She began menstrual periods at the age of 13 and has regular cycles of approximately 28 days. Secondary sexual characteristics include large breasts, axillary, genital, and leg hair, and axillary apocrine gland function.

\section{Family history}

The grandparents of the proband were second cousins, born in Riccia, a small mountain community in south central Italy.

The grandmother (J.4) had 14 pregnancies with ten live births and four spontaneous abortions in the first trimester of pregnancy. Three of the live births, cases II.4, II.9, and II.12, died in the first 7 months of life with congenital heart defects. Case II.9 had a patent foramen ovale, "anterior curvature of the sternum with depression of the epigastrium", and was described as being immature for a term baby. Case II. 12 had a ventricular septal defect.

The grandmother (I.4) died at the age of 49 in 1952 of metastatic adenocarcinoma arising in the greater curvature of the stomach. A teratoma of the left ovary (with hair and teeth-like appendages) was also found on exploratory laparotomy. The grandfather of the proband is well at the age of 76 with mild diabetes mellitus.

Three uncles of the proband are sterile with documented azoospermia, and two (II.7 and II.8) are balanced translocation carriers, while the third (II.3) died accidentally before karyotyping. Case II. 8 also had a partially descended left testis that was surgically removed.
In 1975, at the age of 37, case II.7 developed acute progranulocytic leukaemia, with a peripheral white blood count of 30000 and $90 \%$ blasts. The immature myeloid cells on bone marrow had Auer rods and many azurophilic cytoplasmic granules. Treatment with vincristine, prednisone, cytosine arabinoside, and daunomycin led to a complete remission after a complicated 2-month period in hospital. Additional maintenance therapy with daunomycin was given over an 18-month period and the patient remains in complete clinical remission without therapy. The patient reported no exposure to suspected leukaemogens.

Case II.10, the mother of the proband, had megacolon as a child and suffers from chronic constipation as an adult. Because of irregular menses and infertility of 3 years' duration, culdoscopy was performed and polycystic ovaries found. Prednisone was taken from 2 to 6 months before each of four pregnancies. Her first (III.6) terminated prematurely at 6 months with polyhydramnios and a small, insufficient placenta, despite estinyl oestradiol (Estinyl) and hydroxyprogesterone (Delalutin) for threatened abortion. No congenital anomalies were noted at necropsy. The second (III.7) went to term and is a clinically normal balanced translocation carrier. The third (III.8) is the proband and the fourth pregnancy ended as a complete abortion at one month.

Case II.2, a 53-year-old aunt of the proband, had multiple vocal cord polyps, fibrocystic disease of the breasts, and multiple ovarian cysts noted at partial oophorectomy. She had five spontaneous abortions in the first trimester and carried one infant (III.1) to 7 months taking diethylstilbestrol for threatened abortion. This child (III.1) died with hyaline membrane disease of the lung and had no detectable heart defects or other anomalies at necropsy.

Case 1I.6, another aunt of the proband, had polycystic ovaries, fallopian tube cysts, and endocervical polyps found at panhysterectomy and bilateral salpingo-oophorectomy. She had four pregnancies. Her first child (III.2), a male infant, resembled his younger sister (III.3) with a partial trisomy 18 . He was premature, weighing $1.9 \mathrm{~kg}$ at birth, and had a high arched palate, low set ears, mental retardation, poor muscle tone and co-ordination, seizures, a probable ventricular septal defect, and the mother was told he was blind. He died at 10 months of age. The two other male offspring (III.4 and III.5) were clinically and karyotypically normal.

The karyotypically normal uncle of the proband (II.5) had no medical problems. His wife had seven normal term pregnancies and two first trimester spontaneous abortions. 


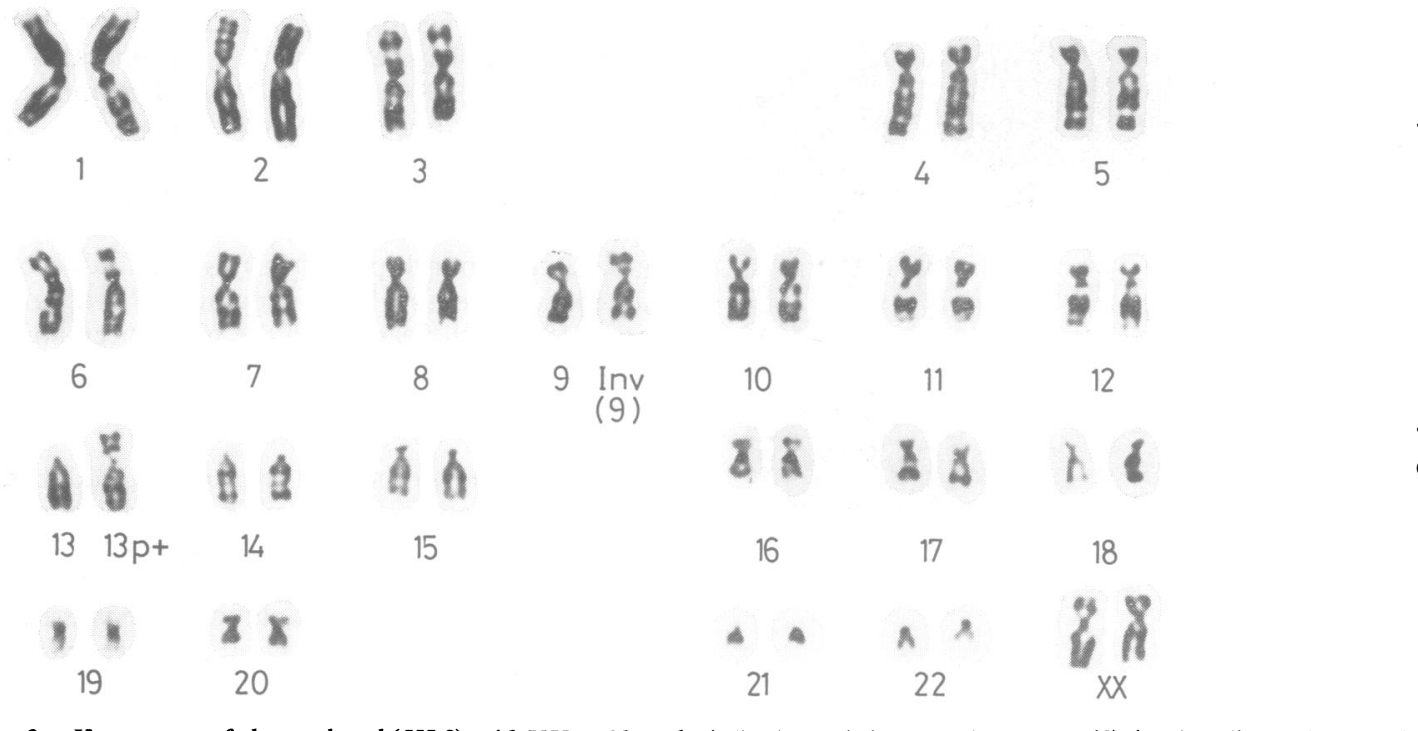

FIG 3 Karyotype of the proband (III.8): 46,XX,-13,+der(13),t(13;18) (p13;q12) mat, inv(9) (p11;q12). (G banding.)

Clinical information on the brothers and sisters of the proband's grandparents shows that none had children or grandchildren with congenital anomalies, infertility, or cancer, and, other than adult onset diabetes and a cerebral vascular accident, none had major medical illnesses.

\section{CYTOGENETIC INVESTIGATIONS}

Both cousins with the partial trisomy 18 phenotype had identical unbalanced translocations with a 13p + chromosome (fig 3). The extra chromosomal material appears to be the distal portion of chromosome 18 translocated onto the short arm of 13 , with the karyotype being $46, X X,-13,+\operatorname{der}(13) t(13 ; 18)(p 13$; q12)mat. Silver staining confirmed that the $13 p+$ chromosome retained the nucleolus organiser region (fig $4 \mathrm{a}$ ), so that the secondary constriction of 13 was included in the translocation chromosome. The proband (III.8) also carried an inverted chromosome 9 (fig 4b) inherited from the father; her karyotype is $46, X X,-13,+\operatorname{der}(13) t(13 ; 18)(p 13 ; q 12) m a t, i n v(9)(p$ $11 ; \mathrm{q} 12) .11$

The mothers of the partial trisomy 18 cases shared identical balanced translocations (fig 5), 46,XX,t $(13 ; 18)(\mathrm{p} 13 ; \mathrm{q} 12)$. The proband's sister (III.7) and a maternal aunt (II.2) also had this karyotype. Two maternal uncles (II.7 and II.8) had the karyotype $46, X Y, t(13 ; 18)(p 13 ; q 12)$. A third uncle (II.5) had a normal karyotype, $46, X Y$.

Chromosome analyses performed on the proband's cousins (III.4 and III.5), grandfather (I.2), and other family members (I.1, I.3, and II.1) were normal.

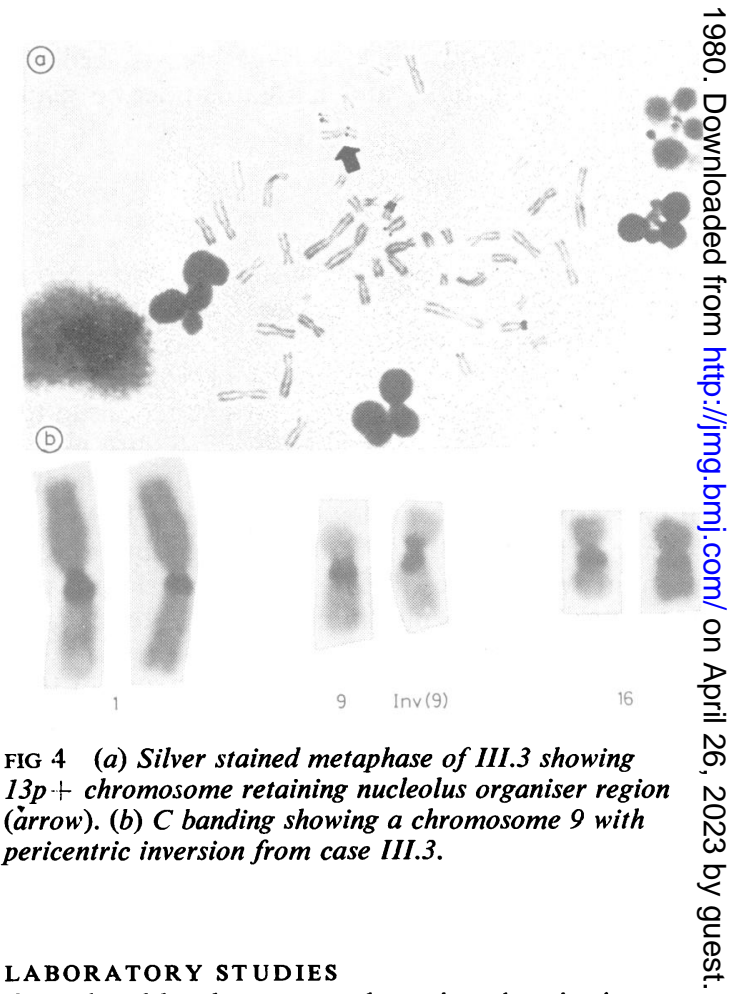

Complete blood counts and routine chemistries were normal, as were gonadotrophin levels in males. $\stackrel{\circ}{\circ}$ Serum immunoglobulins were slightly raised in cases II.7 (IgG), II.2 (IgM), II.6 (IgG), and II.10 (IgG) 
A A
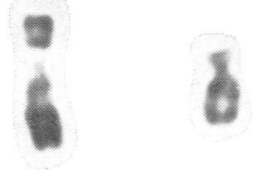

B
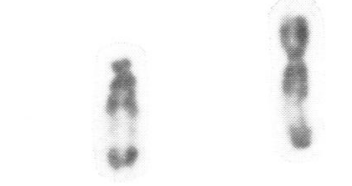

18
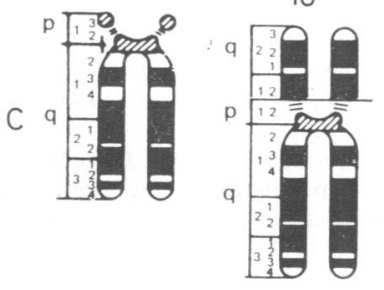

$13 p^{+}$

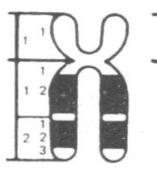

18

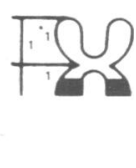

$18 q-$

FIG 5 Chromosome pairs 13 and 18 from case II.10: 46, $X X, t(13 ; 18)(p 13 ; q 12)$. (a) $G$ banding; (b) $R$ banding; (c) schematic representation.

but no monoclonal rises were found on immunoelectrophoresis. Weakly positive rheumatoid factors were found in II.5, II.10, and I.2, but other autoantibody determinations were negative. In vitro immune response to phytohaemagglutinin, Concanavalin $\mathrm{A}$, pokeweed mitogen, staph phage lysate, and mixed leucocyte culture was depressed initially in case II.7, shortly after completion of chemotherapy, but these values later returned to normal. There was no linkage of red blood cell enzymes, red blood cell antigens, or HLA to the presence of the balanced translocation. Translocation carriers II.2, II.7, II.10, and III.7 and karyotypically normal II.5 inherited a 'weak B' variant of acid phosphatase, a marker on chromosome 2. Peptidase A, a red blood cell marker on chromosome 18, was type 1 in all cases, as was esterase D on chromosome 13.

\section{Discussion}

Reciprocal translocations are a well known source of heritable chromosome imbalance in man. ${ }^{12}$ In the family presented here, three generations were probably affected, with imbalance manifest as partial trisomy 18 phenotype. Although the proband and her cousin were the only affected family members

available for karyotyping, the clinical features of another cousin (III.2), two uncles (II.9 and II.12), and an aunt (II.4) suggest that they also had partial trisomy 18 phenotype.

The unbalanced carriers of this family had developmental and mental retardation, failure to thrive, micrognathia, high arched palate, low set malformed ears, congenital eye defects, overlapping fingers, microcephaly, heart defects, hypertrophied clitoris with congenital hypoplastic labia, and hypertonia. These features include many, but not all, of the defects reported in classical trisomy $18 .^{21314}$ The long survival of case III. 3 is exceptional and her normal menstrual and secondary sexual development remarkable. The early deaths of cases II.4, II.9, II.12, and III.2 are more typical for this syndrome. ${ }^{14}$ The tendency for females with trisomy 18 to survive longer than males has been noted. ${ }^{15}$

The grandmother of the proband (I.4) was almost certainly a balanced carrier and, presumably, the first of her generation since none of her sibs or their offspring showed signs of chromosomal dysfunction. In most reported cases, balanced translocation carriers are phenotypically normal. ${ }^{12}$ However, in this family, all female carriers had cystic abnormalities of the ovaries. Cases II.6 and II.10 probably had Stein-Leventhal syndrome based on histological and clinical evidence, and fertility was abnormal in cases II. 2 and II.10.

Male carriers of the balanced translocation were sterile with a few abnormally shaped sperm of absent or limited motility seen on semen analysis. Case II.3 was also sterile and presumably a translocation carrier. A survey of cytogenetic abnormalities in 1599 infertile men identified nine with autosomal translocations, ${ }^{16}$ including one with the karyotype $46, X Y, t(13 ; 18)(q 12 ; q 23)$. In this case, the balanced translocation itself appeared not to be the sole cause of infertility since his father had an identical karyotype. Variable fertility in subjects with balanced translocations may result from pairing disturbances during gametogenesis causing aspermia, preimplantation zygote loss, or miscarriage of the unbalanced fetus. ${ }^{17-19}$ In the present family, abnormalities in gonadal structure or function were suggested by aspermia in males, and polycystic ovaries with infertility in some females. Perhaps some gene(s) controlling reproductive development occurs on the long arm of chromosome 18, with normal function interrupted at the breakpoint. Such a mechanism may also explain the high frequency of cryptorchidism in trisomy 18 males, an abnormality found in one translocation carrier (II.8). ${ }^{13}$ The occurrence of 12 spontaneous abortions in translocation carriers in this family suggests a higher than expected 
incidence, presumably because of rejection of fetuses with lethal chromosome complements. This apparent high frequency contrasts with that reported by Ford and Clegg ${ }^{12}$ who found that spontaneous abortion in translocation heterozygotes was not greater than in the general population.

In the family reported here, one carrier had acute progranulocytic leukaemia and another had adenocarcinoma of the stomach and teratoma. Benign growths (vocal cord polyps and endocervical polyps) were found in two other carriers, suggesting a possible link with neoplasia. In occasional families, balanced translocations have been associated with the development of leukaemia or cancer. ${ }^{20-24}$ In one instance, hereditary renal cell carcinoma was associated in all cases with a balanced translocation between chromosomes 3 and 8 , including several with previously unsuspected tumours. ${ }^{25}$ In the family reported here, the translocation was not linked to a specific type of cancer, although it is possible that an underlying defect could have predisposed both to chromosome abnormalities and cancer. ${ }^{26}$

Although aetiological laboratory studies identified no premalignant abnormalities in family members, the clinical findings suggest a need for more precise laboratory evaluation. Such studies, looking at DNA repair and other aspects of chromosomal function, are currently under way to define a suspected defect that may predispose to the cytogenetic abnormalities and malignancy.

Dr Norman B Schneider, Lakeworth, Florida, successfully treated the uncle of the proband for leukaemia and generously made his office available for interviews and blood drawing. Drs Richard J Warren and Ronald Cantwell, Miami, Florida, assisted in the evaluation of cases I.2, II.5, II.6, II.7, II.8, and III.3 and performed initial karyotypes. Dr Jack $H$ Dean and Frances LeSane, Litton Bionetics Inc, Kensington, Maryland, performed in vitro immune function studies; Dr Paul I Terasaki performed HLA typing; Robin Henson and Deborah McGuire RN assisted with interviews, blood drawing, and data gathering; Drs Louise C Strong, Houston, Texas, Joseph F Fraumeni Jr, and John J Mulvihill, Bethesda, Maryland, offered valuable advice; and Ruth E Brounstein edited and typed the manuscript. Supported in part by NCI Contracts NO1-CP-61011 and NO1-CP-61016, and NIH Grant CA-19834.

\section{Addendum}

One explanation for the coincidence of malignancy and chromosomal abnormalities in this family is that some underlying defect may predispose to both $\stackrel{\mathbb{D}}{\overparen{D}}$ Preliminary results of a new assay of chromosoma? instability show a higher rate of induced chromoso $\overrightarrow{\vec{F}^{2}}$ mal breakage in affected family members than in? controls or unaffected family members. ${ }^{27}$ Further

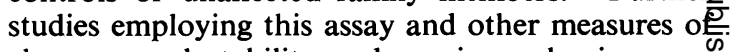
chromosomal stability and repair mechanisms are planned.

\section{References}

1 Jacobs PA, Melville M, Ratcliffe S. A cytogenetic survey of 11,680 newborn infants. Ann Hum Genet 1974;37:359-76

2 Hamerton JF. Human cytogenetics. vol 2. New Yorkẹ̣ Academic Press, 1971.

3 Giblett ER. Genetic markers in human blood. Philadelphia ${ }^{V}$ Lippincott, 1969.

4 Terasaki PI, Bernoco D, Park MS, Ozturk G, Iwaki Y W Microdroplet testing for HLA-A, -B, -C, and -D antigens
Am J Clin Pathol 1978;2:103-20.

5 Dean JH, Connor R, Herberman RB, Silva J, McCoy JL $\overrightarrow{-}$ Oldham RK. The relative proliferation index as a more sensitive parameter for evaluating lymphoproliferative responses of cancer patients to mitogens and alloantigens Int J Cancer 1977;20:359-70.

- Seabright M. A rapid banding technique for human chromosomes. Lancet 1971 ;ii:971-2.

7 Wang HC, Fedoroff S. Banding in human chromosomes treated with trypsin. Nature $1972 ; 235: 52-4$.

8 Scheres JMJC. Production of $\mathrm{C}$ and $\mathrm{T}$ bands in humaro chromosomes after heat treatment at high $\mathrm{pH}$ and staining with 'stains-all'. Hum Genet 1974;23:311-4.

9 Craig-Holmes AP, Moore FB, Shaw MW. Polymorphism of human C-band heterochromatin. I. Frequency of variants. Am J Hum Genet 1973;25:181-92.

10 Varley JM. Patterns of silver staining of human chromo somes. Chromosoma 1977;61:207-14.

11 Hansmann I. Structural variability of human chromosome 9 in relation to its evolution. Hum Genet 1976;31:247-62

12 Ford CE, Clegg HM. Reciprocal translocations. $\mathrm{Br} \mathrm{Me} \not$. Bull 1969;25:110-4.

13 Hodes ME, Cole J, Palmer CG, Reed T. Clinical experi ence with trisomies 18 and $13 . J$ Med Genet $1978 ; 15: 48-60^{\circ}$

14 Taylor AI. Autosomal trisomy syndromes: a detailed study of 27 cases of Edwards' syndrome and 27 cases of. Patau's syndrome. Am J Med Genet 1968;5:227-52.

15 Weber WW. Survival and sex ratio in trisomy 17-18? Am J Hum Genet 1967;19:369-77.

16 Chandley AC, Edmond P, Christie S, Cytogenetics and infertility in man. I. Karyotype and seminal analysis. Ann Hum Genet 1975;39:231-54.

17 Chandley AC, Christie S, Fletcher J, Frackiewicz A Jacobs PA. Translocation heterozygosity and associated subfertility in man. Cytogenet Cell Genet 1972;11:516-33;

18 Lucas M, Wallace I, Hirschhorn K. Recurrent abortions and chromosome abnormalities. Br J Obstet Gynaeco $N$ 1972;79:1119-27.

19 Léonard C, Bisson JP, David G. Male sterility associateck with familial translocation heterozygosity: $t(8 ; 15)^{\circ}$ (q22;p11). Arch Androl 1979;2:269-75.

20 Knudson AG Jr. Genetics and etiology of human cancer Adv Hum Genet 1977;8:1-66.

21 Garson OM, Milligan WJ. Acute leukaemia associate ${ }^{6}$ with an abnormal genotype. Scand J Haematol 1974;12 256-62. 
22 Hinkes E, Crandall BF, Weber F, Craddock CG. Acute leukemia with C-G chromosome translocation. Blood $1973 ; 41(2): 259-63$.

23 Goh K. Smaller G(Gp-) and $(G p-; D p+)$ chromosomes : a familial study with one member having acute leukemia. Am J Dis Child 1968;115:732-8.

24 Riccardi VM, Humbert JR, Peakman D. Acute leukemia associated with trisomy 8 mosaicism and a familial translocation 46,XY,t(7;20) (p13;p12). Am J Med Genet $1978 ; 2(1): 15-21$.

${ }^{25}$ Cohen AJ, Li FP, Berg S, et al. Hereditary renal-cell carcinoma associated with a chromosomal translocation. $N$ Engl J Med 1979;301:592-5.

26 Zuelzer WW, Thompson RI, Mastrangelo R. Evidence for a genetic factor related to leukemogenesis and congenital anomalies: chromosomal aberrations in pedigree of an infant with partial D trisomy and leukemia. $J$ Pediatr $1968 ; 72(3): 367-76$.

27 Hsu TC, Au WW, Strong LC, Johnston DA. A shortterm cytogenetic test for genetic instability in humans. Proceedings of the International Workshop on Short-term Tests for Chemical Carcinogens, Vancouver, British Columbia, August 1979 (in press).

Requests for reprints to Dr William A Blattner, Environmental Epidemiology Branch, National Cancer Institute, Landow Building, Room 3C-07, Bethesda, Maryland 20205, USA. 